\title{
CLINICAL VALIDATION OF THE ANSI C63.19 DRAFT STANDARD FOR MEASURING COMPATIBILITY BETWEEN DIGITAL WIRELESS PHONES AND HEARING AIDS
}

\author{
Robert E. Schlegel, Hank Grant, and Tamy L. Fry \\ Center for the Study of Wireless Electromagnetic Compatibility \\ School of Industrial Engineering \\ University of Oklahoma \\ Norman, OK 73019
}

\begin{abstract}
Acoustic interference can be generated in hearing aids by the pulsed transmission signal of a digital wireless phone. This interference, resembling a buzzing, clicking, or static sound, is annoying and can seriously degrade the intelligibility of the speech. The objective of the ANSI C63.19 Draft Standard is to provide a simple, reliable test procedure for measuring the immunity of hearing aids to this interference. To clinically validate the standard, hearing aids were custom manufactured for eighteen hearingimpaired participants. The participants rated the effects of the interference experienced when using five digital wireless phone technologies (CDMA at 800 and $1900 \mathrm{MHz}$, TDMA-50 Hz at 800 and $1900 \mathrm{MHz}$, and TDMA-217 $\mathrm{Hz}$ at $1900 \mathrm{MHz}$ ) at five transmission power levels $(0,6,12,18$, and $24 \mathrm{dBm})$. More than two-thirds of the subjects responded as predicted by acoustic measurements of the interference. The remaining subjects experienced difficulties unrelated to wireless phone interference due to severe hearing loss or excessive feedback. These results support the use of acoustic measurements of immunity as the basis for the ANSI C63.19 standard.
\end{abstract}

\section{BACKGROUND}

Acoustic interference can be generated in hearing aids by the pulsed transmission signal of a digital wireless phone. This interference, resembling a buzzing, clicking, or static sound, is annoying and can seriously degrade the intelligibility of the speech. Thus, many hearing aid wearers are denied access to the benefits of digital wireless technology. In April 1996, ANSI Accredited Standards Committee C63 established a task group under its subcommittee on medical devices ( $\mathrm{SC} 8$ ) to develop a standard documenting the methods of measurement and defining the limits for electromagnetic compatibility between wireless phones and hearing aids. In 1999, the committee provided a draft document C63.19-xxxx, American National Standard for Methods of Measurement of Compatibility between Wireless Communications Devices and Hearing Aids. The objective of the ANSI C63.19 Draft Standard is to provide a simple, reliable test procedure for measuring the immunity of hearing aids to electromagnetic interference. The C63.19 acoustic measurement procedure results in an immunity rating for the hearing aid at both the low (800-950 MHz) and the high (1800-1900 MHz) carrier frequency bands.

In previous work, the University of Oklahoma Center for the Study of Wireless Electromagnetic Compatibility conducted an acoustic measurementbased evaluation of the standard (commonly referred to as the objective study). The purpose of the current research was to determine correspondence of hearing aid immunity and wireless phone emissions measured according to the standard with actual interference obtained with specific combinations of hearing aids and phones. This work will ultimately lead to the creation of user guides to assist hearing aid wearers in purchasing and using wireless phones without interference.

The overall objective of the current research was to relate subjective ratings of speech intelligibility, usability, and annoyance to acoustic 
measurements of the interference between wireless phones and hearing aids. The C63.19 subjective validation study was subdivided into two parts: (1) subjective ratings of speech intelligibility, annoyance, and usability, and (2) objective acoustic measurements of hearing aid response.

\section{METHODOLOGY}

Hearing aids were custom manufactured for eighteen hearing-impaired participants. The aids spanned a range of immunity levels from no interference to severe interference. All participants were patients of the Hough Ear Institute in Oklahoma City, Oklahoma, which provided assistance in subject recruiting, audiometry testing, hearing aid fitting and follow-up. The participants were chosen to represent a range of hearing loss configurations and hearing loss severity. Approval for human subject testing was obtained from the University of Oklahoma Institutional Review Board - Norman Campus.

Each participant rated the effects of the interference experienced when using five digital wireless phone technologies (CDMA at 800 and $1900 \mathrm{MHz}$, TDMA-50 Hz at 800 and $1900 \mathrm{MHz}$, and TDMA-217 Hz at $1900 \mathrm{MHz}$ ) at five transmission power levels $(0,6,12,18$, and 24 $\mathrm{dBm})$. The TDMA-217 Hz phones were operated via a base station simulator that controlled phone power and transmitted a recorded conversation to the phone.

\section{Subjective Testing}

The subjective testing involved ratings and perceptions of speech intelligibility ( 1 to 5 representing "Completely Intelligible" to "Unintelligible" plus an indication of the proportion of words understood), usability (1 to 4 representing "Highly Usable" to "Not Usable"), and annoyance $(0$ to 5 representing "No Interference" to "Unbearable"). The intelligibility and usability scales were used only in the TDMA- $217 \mathrm{~Hz}$ phone tests in which speech was injected into the phone. These scales are the same as those used by Levitt and Kozma-Spytek (1999) for similar research on wireless phone interference. The annoyance scale was used in all phone tests.

\section{Acoustic Measurements}

In order to relate the subjective ratings to the actual interference, acoustic measurements were taken to determine the response of each hearing aid to three phones (CDMA at $800 \mathrm{MHz}, \mathrm{CDMA}$ at $1900 \mathrm{MHz}$, and TDMA-50 Hz at $1900 \mathrm{MHz}$ ). Six acoustic measurements were taken for each of the three phones. Interference measurements were made at each of the five power levels used in the subjective testing, plus an ambient measurement with the phone on but not transmitting.

A B\&K 2144 Real-Time Frequency Analyzer was used to measure the hearing aid response during the objective tests of interference. The hearing aid was connected to the B\&K Frequency Analyzer through a $50 \mathrm{~cm}$ length of Tygon tubing that was threaded through the side of an acoustic test chamber whose walls were covered with acoustic foam to reduce the ambient noise level. The angle of the hearing aid with respect to the wireless phone was adjusted to obtain a "typical use" position.

\section{Experimental Procedure}

Each participant was tested only with the hearing aid custom-manufactured and fit for the purposes of this research. The total testing time required for each participant was approximately two hours. A balanced test sequence was used to minimize any bias due to trial ordering. Trials were varied by phone unit and power level according to a modified Latin Square theme such that each participant was tested using a unique phone by power level ordering. To address test-retest reliability, each participant performed the test sequence twice.

Participants were instructed to set the gain of the hearing aid to the "most comfortable level" (MCL) while listening to speech through a TDMA$217 \mathrm{~Hz}$ phone at low RF power. After the gain of the hearing aid had been adjusted, the researcher recorded the participant's responses to ensure that the participant had heard and understood the conversation. All hearing aids were tested in the microphone (M) mode. Five participants whose hearing aids had telecoil capability performed one additional test sequence in telecoil (T) mode. 
To simulate "actual use" conditions, each participant was allowed to determine the exposure time, separation distance, and orientation of the phone with respect to the hearing aid. During testing, the researchers verified that each participant employed a realistic phone-use scenario with respect to distance and orientation. Participants were instructed to allow sufficient exposure time to provide an accurate estimation of the annoyance and of the effects on speech intelligibility.

\section{RESULTS}

Based on the acoustic measurements with three wireless phones, the aids for six participants demonstrated little or no interference at either 800 $\mathrm{MHz}$ or $1900 \mathrm{MHz}$. The data for one of these participants was not usable due to a moderately severe hearing loss coupled with substantial feedback from the hearing aid, making the phones unusable. Unrelated to wireless phone RF interference, this person had difficulty understanding any speech through the phone. The remaining five of these six participants appropriately reported no annoying interference and $100 \%$ speech intelligibility.

For the remaining twelve aids, there was often a difference in the level of immunity between the two RF carrier frequency bands. The aids showing interference were categorized as producing either moderate or severe interference in each band. In general, the level of correspondence between the subjective ratings and the measured interference was primarily a function of the immunity of the aid.

Seven participants demonstrated high correspondence between the ratings and the interference as a function of changes in phone power level. These participants experienced moderate to severe interference in their aids at either $800 \mathrm{MHz}$ or $1900 \mathrm{MHz}$ or both. Three of these aids had telecoil capability. In this mode, interference was greater and seriously affected speech intelligibility and annoyance. Another participant, whose aid produced minimal interference in microphone mode and substantial interference in telecoil mode, provided constant ratings across power levels when tested in microphone mode and appropriately poor ratings when in telecoil mode. Another participant, whose aid produced significant interference in both modes at $1900 \mathrm{MHz}$, had a severe hearing loss and was not able to provide usable data due to poor speech intelligibility and high feedback with all phones.

The final three participants demonstrated little or no change in rating response as a function of power level. This was believed to be due to low levels of interference at $800 \mathrm{MHz}$ or $1900 \mathrm{MHz}$ (but not both) or high levels of feedback as the phone is coupled to the ear.

\section{CONCLUSIONS}

In summary, thirteen of the eighteen participants responded as predicted by the acoustic measurements made with actual wireless phones. The remaining participants experienced difficulties unrelated to wireless phone interference (severe hearing loss or excessive feedback). While all aids having a high immunity. classification based on measurements made according to the C63.19 standard were included in this group, it is important to point out that other aids with a lower immunity classification also performed well. This points to the need for further refinement of the immunity measurement and classification procedures.

A proposed alternative to the existing C63.19 performance criteria and categories involves the use of only three categories representing "Poor Performance," "Good Performance," and a transition category of "Intermediate Performance." The proposed spread between the category limits distinguishing "Poor Performance" from "Good Performance" is $10 \mathrm{~dB}$ (field strength measurement for hearing aid immunity or wireless device emission), compared with the original $5 \mathrm{~dB}$ spread.

There are three primary advantages to the use of the proposed categorization scheme:

1. There are fewer classification categories for hearing aids and for wireless devices. This simplifies the assignment process and the system performance classification for manufacturers and consumers.

2. The proposed split points, which employ a $10 \mathrm{~dB}$ spread rather than $5 \mathrm{~dB}$, more accurately reflect the precision that is readily achievable in both the acoustic and subjective measurements (in the absence of any reliability data to the contrary). 
3. The proposed categories provide no worse agreement (and in some cases better agreement) among the C63.19 standard measurements, the measured acoustic data from our laboratory measurements, and the subjective data on annoyance, speech intelligibility, and usability. In other words, there is no loss in predictive accuracy by moving to the proposed categorization scheme using only three categories.

\section{SUMMARY}

In summary, the results of this study support the use of acoustic measurements of immunity as the basis for the ANSI C63.19 standard. The results also demonstrate the existence of hearing aids that can be used with success with a number of digital phones. Six of the eighteen aids demonstrated no interference or very slight interference at the highest power level when used with both $800 \mathrm{MHz}$ and $1900 \mathrm{MHz}$ phones. These conclusions provide an encouraging forecast of substantially improved access to digital wireless service by those individuals with impaired hearing.

\section{ACKNOWLEDGMENTS}

The EMC Center gratefully acknowledges the project funding and direction provided by supporters of the ANSI C63.19 effort, in particular, Ericsson, Motorola, Nokia, QuAlCoMM, and Siemens Business Communications. The Center also recognizes the ongoing support of the following additional sponsors: AirTouch Communications, AT\&T Wireless Services, BellSouth Cellular, Cellular Telecommunications Industry Association, Hewlett-Packard, Lucent Technologies, Medtronic, Northern Telecom, Personal Communications Industry Association, PrimeCo Personal Communications, and SBC Wireless. Frye Electronics is acknowledged for their generous loan of a FONIX ${ }^{\circledR} 6500$-CX for use throughout the $\mathrm{C} 63.19$ validation work. Additional thanks are extended to EMC Center Research Associate Glenn Kuriger and Graduate Research Assistant Dorene Seah, for invaluable assistance during the testing phase of the study. The Center appreciates the support of Dr. Kenneth Dormer of the Hough Ear Institute, which provided study participants under subcontract to the Center, and Perma Scates-Jessup for her numerous hours spent in subject recruiting and follow-up, audiometry testing, and hearing aid fitting.

\section{REFERENCES}

American National Standards Institute (ANSI S3.22 - 1987). Specification of hearing aid characteristics. New York, NY: Acoustical Society of America.

American National Standards Institute (C63.191999). Methods of measurement of compatibility between wireless communications devices and hearing aid. New York, NY: IEEE.

Levitt, H., and Kozma-Spytek, L. (1999). Signalto-interference ratios associated with usability and intelligibility ratings. Presentation to a meeting of the C63.19 Working Group, Boca Raton, Florida, April 27, 1999.

Schlegel, R.E., Srinivasan, S., Grant, H., Shehab, R.L., and Raman, S. (1998). Clinical assessment of electromagnetic compatibility of hearing aids and digital wireless phones. In Proceedings of the 42nd Annual Meeting of the Human Factors and Ergonomics Society. Santa Monica, CA: Human Factors and Ergonomics Society.

Schlegel, R.E., Fry, T.L., and Grant, H. (1999). Investigation of the interaction between wireless phones and hearing aids, Phase III-B: Subjective validation study. Norman, OK: Center for the Study of Wireless Electromagnetic Compatibility, University of Oklahoma. 